\title{
ON CONTINUOUS CURVES IN $n$ DIMENSIONS*
}

\author{
BY G. T. WHYBURN AND W. L. AYRES $†$
}

If $M_{1}$ and $M_{2}$ are subsets of a connected point set $M$, the subset $K$ of $M$ is said to separate $M_{1}$ and $M_{2}$ in $M$ if $M-K$ is the sum of two mutually separated sets containing $M_{1}$ and $M_{2}$ respectively. R. L. Moore $\ddagger$ has shown that in order that a plane continuum $M$ be a continuous curve $\S$ it is necessary and sufficient that for every two distinct points $A$ and $B$ of $M$ there should exist a subset of $M$ which consists of a finite number of continua and which separates $A$ and $B$ in $M$. Consider the following example: Let $S_{i}(i=1,2)$ be the set of all points $(x, y, z)$ in three dimensions such that $x=(-1)^{i}$, $-1 \leqq y \leqq 1,0 \leqq z \leqq 1$. Let $R_{0}$ be the set of all points $(x, y, z)$ such that $-1 \leqq x \leqq 1,-1 \leqq y \leqq 1, z=0$. For each integer $n>0$, let $R_{n}$ be the set of all points $(x, y, z)$ such that $-1 \leqq x \leqq 1$, $-1 \leqq y \leqq 1, z=1 / n$. Let

$$
M=S_{1}+S_{2}+\sum_{n=0}^{\infty} R_{n} .
$$

It is easy to see that every two points of $M$ may be separated by a single subcontinuum of $M$ and yet $M$ is not a continuous curve. Hence the condition given by Moore is not sufficient in order that a continuum in $n$ dimensions $(n>2)$ be a continuous curve. In this paper we give two modifications (Theorems 2 and 4) of Moore's theorem which hold in $n$ dimensions.

* Presented to the Society, October 29, 1927.

$\dagger$ National Research Fellow in Mathematics.

$\ddagger A$ characterization of a continuous curve, Fundamenta Mathematicae, vol. 7 (1925), pp. 302-307.

$\S$ We shall use the term continuous curve in the sense of a point set which is closed, connected and connected im kleinen. See R. L. Moore, Concerning simple continuous curves, Transactions of this Society, vol. 21 (1920), p. 347. 
THEOREM 1.* If $M$ is a continuous curve in euclidean space of $n$ dimensions, $K$ is a bounded subcontinuum of $M$ and $\epsilon$ is any positive number, then there exists a set $L$ such that (1) $K+L$ is a continuous curve which is a subset of $M$, (2) every point of $L$ is within a distance $\epsilon$ of some point of $K$, (3) $L$ consists of a countable set of arcs of $M$, not more than a finite number of which are of diameter greater than any given positive number, (4) $L+K$ is non-dense at every point except those points at which $K$ fails to be non-dense.

Proof. The set $M$ is uniformly connected im kleinen over the set $K$. $\dagger$ Let $\delta_{1}, \delta_{2}, \delta_{3}, \cdots$ be a sequence of positive numbers such that every two points of $K$ whose distance from one another is less than $\delta_{m}$ can be joined by an arc of $M$ whose diameter is less than $\epsilon / 2 m$. For each point $p$ of $K$ and each positive integer $n$, let $C_{n p}$ and $C_{n p}^{\prime}$ be hyperspheres with center $p$ and radii $\epsilon / n$ and $\epsilon /(2 n)$ respectively. $\ddagger$ By the Borel theorem, for each value of $n$ there is a finite subset of the set $\left[C_{n p}^{\prime}\right]$,

$$
C_{n p_{n 1}}^{\prime}, C_{n p_{n 2}}^{\prime}, C_{n p_{n 3}}^{\prime}, \cdots, C_{n p_{n n^{\prime}}}^{\prime},
$$

such that every point of $K$ is in the interior of one of the sets $C_{n p_{n i}}^{\prime}$ for $1 \leqq i \leqq n^{\prime}$. Since $M$ is a continuous curve there are but a finite number,

$$
M_{n i 1}, M_{n i 2}, M_{n i 3}, \cdots, M_{n i m_{n}},
$$

of the components $\S$ of $M \cdot I\left(C_{n p_{n i}}\right)$ that contain points in the interior of $C_{n p_{n i}}^{\prime}$. For each $n, i$ and $j$, let $\left[K_{n i j}\right]$ be the set of

* This theorem contains as a special case a theorem due to H.M.Gehman, Concerning the subsets of a plane continuous curve, Annals of Mathematics, vol. 27 (1925), pp. 29-46, Theorem 3.

$\dagger$ S. Mazurkiewicz, Sur les lignes de Jordan, Fundamenta Mathematicae, vol. 1 (1920), p. 173.

$\ddagger$ If $p$ is a point and $r$ a positive number, the hypersphere with center $p$ and radius $r$ is the set of all points of the space whose distance from the point $p$ is $r$. If $S$ is a hypersphere, $I(S)$ denotes the interior of $S$.

$\S$ A connected subset of a point set $H$ which is not a proper subset of any connected subset of $H$ is called a component of $H$. 
components of $K \cdot M_{n i j} \cdot I\left(C_{n p_{n i}}^{\prime}\right)$. By the Zermelo postulate*, there exists a set of points $\left[P_{n i j}\right]$ such that each set $K_{n i j}$ contains just one point $P_{n i j}$ and each point $P_{n i j}$ belongs to just one component $K_{n i j}$. In the set $\left[P_{n i j}\right]$ there is a finite subset,

$$
P_{n i j}^{1}, P_{n i j}^{2}, P_{n i j}^{3}, \cdots, P_{n i j}^{k_{1}}, \dagger
$$

such that every point of $\left[P_{n i j}\right]$ is within a distance $\delta_{1}$ of some point of this finite set. There exists an arc $\alpha_{n i j}^{r}\left(1 \leqq r \leqq k_{1}-1\right)$ with end points $P_{n i j}^{r}$ and $P_{n i j}^{r+1}$ and lying wholly in $M_{n i j}$. There exists a finite subset,

$$
P_{n i j}^{k_{1}+1}, P_{n i j}^{k_{1}+2}, \cdots, P_{n i j}^{k_{2}},
$$

of the set $\left[P_{n i j}\right]$ such that every point of $\left[P_{n i j}\right]$ is within a distance $\delta_{2}$ of some point of $P_{n i j}^{1}, P_{n i j}^{2}, \cdots, P_{n i j}^{k_{2}}$. Let $\alpha_{n i j}^{r}$ $\left(k_{1} \leqq r \leqq k_{2}-1\right)$ be an arc of $M_{n i j}$ with end points $P_{n i j}^{r+1}$ and some point of $\sum_{s=1}^{k_{1}} P_{n i j}^{s}$. Continue this process indefinitely except that for $t>n$ we place the additional condition on $\alpha_{n i j}^{r}\left(k_{t} \leqq r \leqq k_{t+1}-1\right)$ that it be of diameter less than $\epsilon /(2 t)$. This can be done since any two points of $K$ whose distance from one another is less than $\delta_{t}$ can be joined by an $\operatorname{arc}$ of $M$ whose diameter is less than $\epsilon /(2 t)$.

For each $n, i$ and $j$, there is a countable set of arcs of $M$, $\alpha_{n i j}^{1}, \alpha_{n i j}^{2}, \alpha_{n i j}^{3}, \cdots$, such that (a) each lies interior to a hypersphere of radius $\epsilon / n$ with a point of $K$ as center, (b) only a finite number are of diameter greater than a given positive number, and (c) each has its end points on $K$. For each value of $n$ the numbers $i$ and $j$ range over finite sets of values; hence the set of all $\operatorname{arcs}\left[\alpha_{n i j}^{r}\right]$ for a fixed value of $n$ satisfy conditions (a), (b), and (c) above. And since all arcs $\left[\alpha_{n i j}^{r}\right]$ for a fixed value of $n$ are of diameter less than $2 \epsilon / n$, the set of all arcs $\left[\alpha_{n i j}^{r}\right]$ for all values of $n$ satisfies the condi-

*E. Zermelo, Untersuchung über die Grundlagen der Mengenlehre, Mathematische Annalen, vol. 65 (1908), pp. 261-281.

$\dagger$ The symbol $k_{1}$ denotes a positive integer whose value depends on $n$, $i$ and $j$. 
tion that only a finite number are of diameter greater than a given positive number. Let

$$
L=\sum \alpha_{n i j}^{r}, \begin{array}{ll}
1 \leqq i \leqq n^{\prime}, & 1 \leqq j \leqq m_{n}, \\
1 \leqq r<\infty, & 1 \leqq n<\infty .
\end{array}
$$

We have shown that $L$ satisfies conditions (2) and (3) of our theorem. It remains to prove that (1) and (4) are satisfied. Since only a finite number of the arcs of $L$ are of diameter greater than a given positive number and each has a point on the closed set $K$, every limit point of $L$ which does not belong to $L$ belongs to $K$. Thus $K+L$ is closed. Let $P$ be any point of $K+L$. If $P$ does not belong to $K$ it is easy to see that $K+L$ is connected im kleinen at $P$, for the interiors of hyperspheres of sufficiently small radii and center $P$ contain no point of $K$ and points of only a finite number of $\operatorname{arcs}$ of $L$. If $P$ is a point of $K$ and $\eta$ is any positive number, there is a hypersphere $C_{n p_{n i}}$ which lies entirely in the interior of the hypersphere with radius $\eta / 4$ and center $P$ and such that $I\left(C_{n p_{n i}}^{\prime}\right)$ contains $P$. Let $M_{n i j}$ be the component of $M \cdot I\left(C_{n p_{n i}}\right)$ containing $P$. There exists a positive number $\gamma$ such that every point of $K$ whose distance from $P$ is less than $\gamma$ lies in $M_{n i j}$. There exists a number $\rho>0$ such that every point $p^{\prime}$ of $L$ whose distance from $P$ is less than $\rho$ lies on an arc $\alpha_{p^{\prime}}$ of $L$, one of whose points $e$ belongs to $K \cdot M_{n i j}$ and such that the subarc $p^{\prime} e$ of $\alpha_{p^{\prime}}$ is of diameter less than $\eta / 2 .^{*}$ Let $\sigma$ be the smaller of $\gamma$ and $\rho$. Now let $Q$ be any point of $K+L$ whose distance from $P$ is less than $\sigma$. If $Q$ belongs to $K$ it belongs to $M_{n i j}$. By the method of

* If $S_{\gamma}$ and $S_{d}$ denote hyperspheres with center $P$ and radii $\gamma$ and $d$ respectively, then only a finite number of arcs of $L$ have points in $I\left(S_{d}\right)$ for any $d<\gamma$ and contain no point of $I\left(S_{\gamma}\right) \cdot K$ since any such arc is at least of diameter $\gamma-d$. There is a number $d_{1}>0$ such that for $d \leqq d_{1}$ there is no such arc. Also there is a number $d_{2}>0$ such that no arc of $L$ of diameter greater than $\eta / 2$ contains a point whose distance from $P$ is less than $d_{2}$ unless the arc contains $P$. On each of the finite set of $\operatorname{arcs}$ of $L$ of diameter greater than $\eta / 2$ that contain $P$ there is a point $q$ such that the subarc $q P$ of the arc is of diameter less than $\eta / 2$. Let $d_{3}$ be the smallest of the finite set of distances from $P$ to the points $q$. Let $\rho$ be the smallest of the numbers $d_{1}, d_{2}$ and $d_{3}$. 
construction of $L$, there is a subset $L^{\prime}$ of $L$ such that $M_{n i j}$ contains $L^{\prime}$ and $L^{\prime}+K \cdot M_{n i j}$ is connected. But every point of $L^{\prime}+K \cdot M_{n i j}$ is at a distance from $P$ less than $\eta / 2$ and $L^{\prime}+K \cdot M_{n i j}$ contains both $P$ and $Q$. If $Q$ is not a point of $K$, it lies on an arc $\alpha_{Q}$ of $L$ which contains a point $e$ of $K \cdot M_{n i \text {, }}$ such that the subarc $e Q$ of $\alpha_{Q}$ is of diameter less than $\eta / 2$. Then $\alpha_{Q}+L^{\prime}+K \cdot M_{n i j}$ is a connected subset of $L+K$ containing $P$ and $Q$ and such that every point is at a distance from $P$ less than $\eta$. Therefore $K+L$ is connected im kleinen at every point $P$.

Let $P$ be any point of $K$ at which $K$ is non-dense. Then if $S_{1}$ is any hypersphere with center $P$, the set $I\left(S_{1}\right)$ contains a hypersphere $S_{2}$ such that $S_{2}+I\left(S_{2}\right)$ contains no point of $K$. Since only a finite number of the arcs of $L$ are of diameter greater than a given positive number, there are only a finite number of arcs of $L$ that have points in $I\left(S_{2}\right)$. Then there is a hypersphere $S_{3}$ lying in $I\left(S_{2}\right)$ such that $I\left(S_{3}\right)$ contains no point of $L$. Then the interior of every hypersphere $S_{1}$ with center at $P$ contains a hypersphere $S_{3}$ such that $I\left(S_{3}\right)$ contains no point of $K+L$. Hence $K+L$ is non-dense at the point $P$.

THEOREM 2. In order that a continuum M lying in euclidean space of $n$ dimensions be a continuous curve it is necessary and sufficient that for every two distinct points $A$ and $B$ of $M$ there should exist a subset of $M$ which consists of a finite number of continuous curves and which separates $A$ and $B$ in $M$.

Proof. The condition is necessary. Let $d$ be the distance from $A$ to $B$. Let $S_{1}$ and $S_{2}$ be hyperspheres with center $A$ and radii $d / 2$ and $d / 4$ respectively. Let $H=S_{1}+I\left(S_{1}\right)-I\left(S_{2}\right)$. The set $M \cdot H$ is closed and it is easy to see that there is at least one component of $M \cdot H$ containing points on both $S_{1}$ and $S_{2}$. As $M$ is a continuous curve there cannot be more than a finite number of such components. Let $K_{1}, K_{2}$, $K_{3}, \cdots, K_{m}$ denote the set of all components of $M \cdot H$ which contain a point on $S_{1}$ and a point on $S_{2}$. By Theorem 1, for each $i, 1 \leqq i \leqq m$, there is a continuous curve $M_{i}$ which contains 
$K_{i}$, is a subset of $M$ and such that every point of $M_{i}$ is within a distance $d / 8$ of some point of $K_{i}$. Suppose that $A$ and $B$ lie in a connected subset of $M-\sum_{i=1}^{m} M_{i}$. Then there is an arc with end points $A$ and $B$ lying in $M-\sum_{i=1}^{m} M_{i}{ }^{*}$ This arc contains a subarc $\alpha$ which is a subset of $H$ and has one end point on $S_{1}$ and the other on $S_{2}$. Then $\alpha$ must belong to some set $K_{i}$ and thus to $\sum_{i=1}^{m} M_{i}$. But this is impossible, for $M-\sum_{i=1}^{m} M_{i}$ contains $\alpha$. Therefore $\sum_{i=1}^{m} M_{i}$ separates $A$ and $B$ in $M$.

The condition is sufficient. If $M$ is not a continuous curve there exist two concentric hyperspheres $S_{1}$ and $S_{2}$ and an infinite set of subcontinua $M_{\infty}, M_{1}, M_{2}, M_{3}, \cdots$ of $M$ satisfying the conditions of the Moore-Wilder lemma. $\dagger$ Let $S_{3}$ and $S_{4}$ be distinct hyperspheres concentric with $S_{1}$ and lying between $S_{1}$ and $S_{2}$. Each continuum $M_{i}$ contains a subcontinuum $K_{i}$ which contains a point $P_{i}$ on $S_{3}$ and a point $Q_{i}$ on $S_{4}$ and is a subset of the set $G$ consisting of $S_{3}$ and $S_{4}$ and all points which lie between $S_{3}$ and $S_{4}$. There exists a sequence of integers $n_{1}, n_{2}, \cdots$, such that $\left[P_{n_{i}}\right]$ has a sequential limit point $A$ and $\left[Q_{n_{i}}\right]$ has a sequential limit point $B$. By hypothesis there exists a finite set of continuous curves $C_{1}, C_{2}, C_{3}, \cdots, C_{m}$ which are subsets of $M$ and separate $A$ and $B$ in $M$.

CASE I. Suppose infinitely many of the continua $K_{n_{i}}$ contain a point of $\sum_{k=1}^{m} C_{k}$. As there are but a finite number of the curves $C_{k}$, one curve $C_{k^{\prime}}$ must contain a point $p_{n_{i}}$ of $K_{n_{i}}$ for infinitely many values of $i$. The set $\left[p_{n_{i}}\right]$ has a limit point $P$, which must belong to $M_{\infty}$ and to $G$. Let $\epsilon$ be a positive number such that no point of $S_{1}+S_{2}$ is within a distance $\epsilon$ of $P$. As $C_{k^{\prime}}$ is a continuous curve, the point $P$

* R. L. Moore, Concerning continuous curves in the plane, Mathematische Zeitschrift, vol. 15 (1922), pp. 254-260. Moore's theorem is stated for two dimensions, but the extension to $n$ dimensions is obvious.

$\dagger \mathrm{R}$. L. Moore, Report on continuous curves from the viewpoint of analysis situs, this Bulletin, vol. 29 (1923), p. 296; R. L. Wilder, Concerning continuous curves, Fundamenta Mathematicae, vol. 7 (1925), p. 371. The lemma holds equally well for $n$ dimensions and for unbounded continua. 
belongs to $C_{k^{\prime}}$ and there is a number $\delta_{\epsilon}>0$ such that any point of $C_{k^{\prime}}$ whose distance from $P$ is less than $\delta_{\epsilon}$ can be joined to $P$ by an arc of $C_{k^{\prime}}$ of diameter less than $\epsilon$. There is a point $p_{n_{r}}$ of $\left[p_{n_{i}}\right]$ whose distance from $P$ is less than $\delta_{\epsilon}$. Let $\alpha$ denote an arc of $C_{k^{\prime}}$ with end points $P$ and $p_{n_{r}}$ and of diameter less than $\epsilon$. The arc $\alpha$ contains a point of $M_{n_{r}}$ and a point of $M_{\infty}$ and lies entirely between $S_{1}$ and $S_{2}$. By the Moore-Wilder lemma, $M_{n_{r}}$ is a component of the common part of $M$ and the set composed of $S_{1}$ and $S_{2}$ and all points lying between $S_{1}$ and $S_{2}$. Hence $M_{n_{r}}$ contains the arc $\alpha$. But this contradicts the condition of the lemma that $M_{n_{r}}$ and $M_{\infty}$ have no common points.

Case II. Suppose only a finite number of the continua $K_{n_{i}}$ contain points of $\sum_{k=1}^{m} C_{k}$. The set $M-\sum_{1}^{m} C_{k}$ is the sum of two mutually separated sets $M_{A}$ and $M_{B}$ containing $A$ and $B$ respectively. Every set $K_{n_{i}}$ which contains no point of $\sum_{1}^{m} C_{k}$ lies wholly in $M_{A}$ or wholly in $M_{B}$. There is an integer $j$ such that for $i \geqq j$, the continuum $K_{n_{i}}$ contains no point of $\sum_{1}^{m} C_{k}$. Both $A$ and $B$ are limit points of the set $\sum_{i=j}^{\infty} K_{n_{i}}$. Either infinitely many of the sets $K_{n_{i}}(i \geqq j)$ belong to $M_{A}$ or infinitely many belong to $M_{B}$. If the first holds then $B$ is a limit point of $M_{A}$; under the second possibility the point $A$ is a limit point of the set $M_{B}$. In either possibility we have a contradiction since $M_{A}$ and $M_{B}$ are mutually separated.

The assumption that $M$ is not a continuous curve leads to a contradiction with the assumed condition in either case. Therefore the condition is sufficient.

It is to be noticed that in the proof of the necessity of the condition in Theorem 2 we showed that the separating continuous curves were bounded. Hence we have the following corollary and theorem.

Corollary. If $A$ and $B$ are points of a continuous curve $M$ lying in euclidean space of $n$ dimensions, there exists a subset of $M$ which consists of a finite number of bounded continuous curves and which separates $A$ and $B$ in $M$. 
THEOREM 3. If $K_{1}$ and $K_{2}$ are any two mutually exclusive and closed point sets, one of which is bounded, then $K_{1}$ lies wholly in a finite number of the complementary domains of $K_{2}$.

Proof. Suppose the contrary is true. Then there exists an infinite sequence $D_{1}, D_{2}, D_{3}, \cdots$ of distinct complementary domains of $K_{2}$ each of which contains at least one point of $K_{1}$. For each positive integer $i$, let $P_{i}$ denote a point of $K_{1}$ belonging to $D_{i}$. Let $H$ denote the set of points $P_{1}+P_{2}+P_{3}+\cdots$ By hypothesis either $K_{1}$ or $K_{2}$ is bounded. If $K_{1}$ is bounded, then $H$ is bounded because $H$ is a subset of $K_{1}$; and if $K_{2}$ is bounded, then since $H$ contains at most one point in the unbounded complementary domain of $K_{2}$, it readily follows that $H$ is bounded. Hence, in any case, $H$ is bounded; and since it is infinite, it must have at least one limit point $P$. Since $K_{1}$ is closed and contains $H$, it must contain the point $P$; and since $K_{1}$ and $K_{2}$ are mutually exclusive, $P$ must belong to some complementary domain $D$ of $K_{2}$. Clearly this is impossible, since $P$ is a limit point of $H$, and not more than one point of $H$ can belong to $D$. Thus the supposition that Theorem 3 is not true leads to a contradiction.

THEOREM 4. In order that a continuum $M$ in a euclidean space $E_{n}$ of $n$ dimensions should be a continuous curve it is necessary and sufficient that every two mutually exclusive, closed, and bounded subsets of $M$ should be separated in $M$ by the sum of a finite number of subcontinua of $M$.

Proof.* The condition is sufficient. For suppose a continuum $M$ satisfies the condition but is not a continuous curve. Then by the Moore-Wilder lemmat it follows that there exist two different concentric hyperspheres $C_{1}$ and $C_{2}$ and a countable infinity of mutually exclusive subcontinua of $M: W, M_{1}, M_{2}, M_{3}, \cdots$ such that (1) if $D$ denotes the

* Compare this proof with that given by R. L. Moore for Theorem 1 of his paper, A characterization of a continuous curve, loc. cit.

$\dagger$ See reference to the Moore-Wilder lemma above. 
$n$-dimensional domain whose boundary is $C_{1}+C_{2}$, then each of these continua contains at least one point on each of the hyperspheres $C_{1}$ and $C_{2}$, and each of them, save possibly $W$, is a component of the set of points $M \cdot\left(D+C_{1}+C_{2}\right)$, and (2) $W$ is the sequential limiting set of the sequence of continua $M_{1}, M_{2}, M_{3}, \cdots$. Let $A$ and $B$ denote the sets of points $W \cdot C_{1}$ and $W \cdot C_{2}$ respectively and, for each positive integer $i$, let $a_{i}$ denote the set of points $M_{i} \cdot C_{1}$ and $b_{i}$ the set $M_{i} \cdot C_{2}$. Since $A$ and $B$ are mutually exclusive, closed, and bounded subsets of $M$, by hypothesis there exists a subset $L$ of $M$ such that (1) $M-L$ is the sum of two mutually separated point sets $M_{a}$ and $M_{b}$ containing $A$ and $B$ respectively, and (2) $L$ is the sum of a finite number of continua $L_{1}, L_{2}, L_{3}, \cdots, L_{m}$. Since neither $A$ nor $B$ has a point in common with $L$, and $A$ contains no point of $M_{b}$ and $B$ contains no point of $M_{a}$, therefore there exist open sets $C_{a}$ and $C_{b}$, containing $A$ and $B$ respectively, such that $C_{a}$ contains no point of $L+M_{b}$ and $C_{b}$ contains no point of $L+M_{a}$. There exists an integer $\delta$ such that, for every $j$ greater than $\delta$, the point set $a_{j}$ lies wholly in $C_{a}$ and the point set $b_{j}$ lies wholly in $C_{b}$. Thus, for every $j$ greater than $\delta, M_{j}$ contains a point of $M_{a}$ and also a point of $M_{b}$. But $M_{j}$ is a subcontinuum of $M$, and every subcontinuum of $M$ which contains a point of each of the sets $M_{a}$ and $M_{b}$ must contain at least one point of $L$. Hence, for every $j$ greater than $\delta$, $M_{j}$ contains a point of $L$, and therefore of some one of the sets $L_{1}, L_{2}, \cdots, L_{m}$. It follows that there exists an integer $g$ and an infinite sequence of distinct positive integers $t_{1}, t_{2}, t_{3}, \cdots$ such that, for every $j, L_{g}$ contains at least one point in common with $M_{t_{j}}$. Since, for every $j$, the subcontinuum $L_{g}$ of $M$ contains a point of $M_{t_{j}}$ and a point of $M_{t_{j+1}}$ it follows by a lemma of R. L. Moore's* that $L_{g}$ must contain a point either of $a_{t_{j}}$ or of $b_{t_{j}}$. Thus there exists an infinite sequence of distinct integers $j_{1}, j_{2}, j_{3}, \cdots$, such that either $L_{g}$ has a point in common with each point set

\footnotetext{
* A characterization of a continuous curve, loc. cit., Lemma 2.
} 
of the sequence $a_{j_{1}}, a_{j_{2}}, a_{j_{3}}, \ldots$, or it has at least one point in common with each point set of the sequence $b_{j_{1}}, b_{j_{2}}$, $b_{j_{3}}, \ldots$. In the first case it readily follows that $A$ contains at least one point of $L_{g}$, and in the second case that $B$ contains at least one point of $L_{g}$. But $A+B$ is a subset of $M-L$. Thus the supposition that $M$ is not a continuous curve leads to a contradiction.

The condition is also necessary. For let $M$ be any continuous curve in $E_{n}$, and let $K_{1}$ and $K_{2}$ be any two mutually exclusive, closed, and bounded subsets of $M$. It follows by Theorem 3 that there exists a finite number $D_{1}, D_{2}, D_{3}, \cdots$, $D_{m}$ of the complementary domains of $K_{2}$ whose sum contains the point set $K_{1}$. For each positive integer $i \leqq m$, let $B_{i}$ denote the boundary of $D_{i}$, let $H_{i}$ be the set of points common to $K_{1}$ and $D_{i}$, and let $4 d_{i}$ be the minimum distance between the closed sets of points $H_{i}$ and $B_{i}$. For each point $P$ of $H_{i}+B_{i}$, let $C_{p}$ denote a hypersphere with $P$ as center and radius $d_{i}$, and let $G_{i}^{\prime}$ be the collection of all the hyperspheres $\left[C_{p}\right]$ for all points $P$ of $H_{i}+B_{i}$. Since $K_{1}+K_{2}$, and hence also $H_{i}+B_{i}$, is bounded, then by the Borel theorem there exists a finite subcollection $G_{i}$ of the hyperspheres of $G_{i}^{\prime}$ such that $H_{i}+B_{i}$ is a subset of the sum $I_{i}$ of the interiors of the collection $G_{i}$. Let $T_{i}$ denote the point set $\left(D_{i}+B_{i}\right)$ $-I_{i} \cdot\left(D_{i}+B_{i}\right)$. Then $T_{i}$ is closed. Let $F_{i}$ denote the sum of all the hyperspheres (not including their interiors) of the collection $G_{i}$ which enclose at least one point of $H_{i}$, and let $N_{i}$ be the sum of all those which enclose at least one point of $B_{i}$. Since the least distance between $H_{i}$ and $B_{i}$ is $4 d_{i}$, and since the radius of each hypersphere of $G_{i}$ is $d_{i}$, it follows that $F_{i}$ and $N_{i}$ are mutually exclusive closed sets whose least distance apart is $>d_{i}$. Let $Q_{i}$ denote the collection of all those maximal connected subsets of $M$ which lie wholly in $T_{i}$ and contain at least one point of each of the sets $F_{i}$ and $N_{i}$. Each element of $Q_{i}$ is a continuum, and since $M$ is a continuous curve, it follows by the Moore-Wilder lemma that $Q_{i}$ has just a finite number of elements. Hence $Q_{i}$ is a finite 
collection of mutually exclusive continua $L_{1 i}, L_{2 i}, L_{3 i}, \cdots$, $L_{n_{i} i}$ which belong to $M$.

Now let $L$ denote the point set $\sum_{i=1}^{m} \sum_{j=1}^{n_{i}} L_{j i}$. Then $L$ is the sum of a finite number of mutually exclusive subcontinua of $M$. Let $M_{a}$ denote the sum of all those components of $M-L$ which contain at least one point of $A$, and let $M_{b}$ denote the point set $M-\left(M_{a}+L\right)$. No point of $B$ belongs to $M_{a}$. For if a point $X$ of $B$ belonged to $M_{a}$, then* $X$ could be joined in $M$ to some point $Y$ of $A$ by an arc which contains no point of $L$, and this arc would contain a subarc $t$ which is a subset of some set $T_{i}$ and which has its end points on $F_{i}$ and $N_{i}$ respectively; and the arc $t$ would necessarily be a subset of some continuum of the collection $Q_{i}$, contrary to the fact that $t$ contains no point of $L$. Therefore $B$ must be a subset of $M_{b}$. Since $M$ is connected im kleinen and $L$ is closed, it readily follows that $M_{a}$ and $M_{b}$ are mutually separated. Hence $M-L$ is the sum of two mutually separated sets $M_{a}$ and $M_{b}$ containing $A$ and $B$ respectively, and therefore $L$ separates $A$ and $B$ in $M$.

THEOREM 5. In order that a continuum $M$ in a space of $n$ dimensions should be a Menger regular curve $\dagger$ it is necessary and sufficient that every two points of $M$ should be separated in $M$ by some finite subset of $M$.

Proof. The condition is sufficient. Let $P$ be any point of $M$ and $\epsilon$ any positive number. Let $C_{1}$ and $C_{2}$ be two distinct hyperspheres each of which has $P$ as center and is of radius less than $\epsilon / 4$. Let $D$ denote the domain between $C_{1}$ and $C_{2}$, and let $K$ denote the set of points common to $D+C_{1}+C_{2}$ and to $M$. Then $K$ is closed. Now by Theorem 2 it follows

* R. L. Moore, Concerning continuous curves in the plane, loc. cit.

$\dagger$ A continuum $M$ is said to be a Menger regular curve provided that for each point $P$ of $M$ and each positive number $\epsilon$ there exists an open subset $T$ of $M$ of diameter less than $\epsilon$ which contains $P$ and whose $M$ boundary is finite. The $M$-boundary of an open subset $T$ of a continuum $M$ is the set of all those points of $M-T$ that are limit points of $T$. See $\mathrm{K}$. Menger, Grundz üge einer Theorie der Kurven, Mathematische Annalen, vol. 95 (1925-1926), pp. 276-306. 
that $M$ is a continuous curve. By hypothesis, for each point $X$ of $K$ there exists a finite subset $N_{x}$ of $M$ which separates $X$ and $P$ in $M$. For each such point $X$, the maximal connected subset $H_{x}$ of $M-N_{x}$ which contains $X$ is an open subset of $M$ which does not contain $P$ and whose $M$-boundary is finite (a subset of $N_{x}$ ). Let $G_{0}$ denote the collection of sets [ $H_{x}$ ] for all points $X$ of $K$. Since $K$ is closed and bounded, then by the Borel theorem the collection $G_{0}$ contains a finite subcollection $G$ which covers $K$. Let $R$ denote the sum of all the point sets of the collection $G$. Then $K$ is a subset of $R$, and $R$ is an open subset of $M$. Furthermore $B$, the $M$-boundary of $R$, is finite, for $R$ is the sum of a finite number of the sets $H_{x}$. Now, supposing that $C_{1}$ is within $C_{2}$, let $A$ denote the set of all those points of $B$ which lie on or within $C_{1}$. Now $R+A$ does not contain $P$, for $P$ belongs to no set $H_{x}$ and to no $N_{x}$. Let $T$ denote the maximal connected subset of $M-A$ which contains $P$. It is readily seen that $T$ must lie within $C_{1}$. Hence the diameter of $T$ is less than $\epsilon$. The $M$-boundary of $T$ is finite, because it is a subset of $A$. Then, since $T$ is an open subset of $M$, it follows that $P$ is a regular point of $M$ and that $M$ is a Menger regular curve.

That the condition is necessary follows at once from the definition of a Menger regular curve.

THEOREM 6. If every two points of a continuum $M$ are separated in $M$ by some finite subset of $M$, then every two mutually exclusive, closed, and bounded subsets of $M$ are separated in $M$ by some finite subset of $M$.

Proof. It follows by Theorem 5 that $M$ is a Menger regular curve. Then by a theorem of Menger's, ${ }^{*}$ it follows that every two mutually exclusive, closed, and bounded subsets of $M$ can be separated in $M$ by some finite subset of $M$.

The University of Texas

\footnotetext{
* Loc. cit., Theorem 12.
} 